\title{
DÉPENDANCE CHIMIQUE POUR L'USAGE DE LA MARIJUANA: DU PLAISIR À LA MALADIE. UN GRAVE PROBLĖME DE SANTÉ PUBLIQUE AU BRÉSIL.
}

\section{ARTICLE ORIGINAL}

DIAS, Amanda de Araújo1', DIAS, Édina Lúcia de Araújo², OLIVEIRA, Ciane Martins de $^{3}$, DENDASCK, Carla Viana ${ }^{4}$, OLIVEIRA, Euzébio de ${ }^{5}$

DIAS, Amanda de Araújo Dias. Et al. Dépendance chimique pour l'usage de la marijuana: du plaisir à la maladie. Un grave problème de santé publique au Brésil. Revista Científica Multidisciplinar Núcleo do Conhecimento. An 06, Ed. 03, Vol. 11, p. 78-86. mars 2021. ISSN: 2448-0959, Lien d'accès: https://www.nucleodoconhecimento.com.br/sante/marijuana, DOI: 10.32749/nucleodoconhecimento.com.br/sante/marijuana

\section{ABSTRAIT}

Introduction : La consommation de marijuana a augmenté à l'échelle mondiale, étant la drogue illicite la plus consommée au monde. On estime qu'entre 167 et 315 millions de personnes âgées de 15 à 64 ans ont consommé des drogues illicites au cours de la dernière décennie. Au Brésil, environ 7,5 millions d'étudiants universitaires, répartis dans environ 2.400 établissements, utilisent l'herbe. Méthode : Une étude descriptive a été conduite basée sur une revue de littérature. La recherche a été effectuée par l'intermédiaire de la base de données Scielo et de la bibliothèque virtuelle de santé (BVS). Résultats et discussion : En ce qui concerne les effets de la marijuana, dans certaines études, des symptômes liés à l'usage

\footnotetext{
${ }^{1}$ Medical Academic au Centre Universitaire de l'Etat du Pará - CESUPA.

${ }^{2}$ Psychologue. Troisième cycle en santé mentale chez Escola Superior da Amazônia - ESAMAZ.

${ }^{3}$ Doctorat en génétique médicale. Professeur et chercheur au Centre universitaire de l'État du Pará (CESUPA).

${ }^{4}$ Théologien, Ph.D. en psychanalyse clinique. II travaille depuis 15 ans avec la méthodologie scientifique (méthode de recherche) dans le cadre de l'orientation de production scientifique des étudiants à la maîtrise et au doctorat. Spécialiste de l'étude de marché et de la recherche axée sur la santé.

${ }^{5}$ Doctorat en médecine/maladies tropicales. Professeur et chercheur à l'Université fédérale du Pará - UFPA.
}

RC: 80475

Disponível em: https://www.nucleodoconhecimento.com.br/sante/marijuana 
récréatif et à l'abus de cette drogue sont signalés. Cependant, au niveau universitaire, les effets de la consommation de marijuana peuvent être divisés en symptômes aigus et chroniques. Les effets aigus sont classés comme l'euphorie, les effets physiques et les effets psychiques tels que la dépression, l'hallucination, l'illusion, la somnolence et les troubles de la concentration et de la mémoire à court terme. Conclusion : Certaines preuves sont démontrées qui peuvent expliquer pourquoi les individus choisissent de fumer de la marijuana. Le plus souvent, les effets aigus de l'euphorie, le plaisir et la relaxation sont souhaités, en revanche, il est observé que les dommages associés à cette utilisation finissent par surmonter les avantages illusoires que le médicament offre, conduisant ainsi au plaisir de devenir une maladie.

Mots-clés: Marijuana, consommation, plaisir, maladie.

\section{INTRODUCTION}

L'habitude de consommer des drogues a été présente et établie dans les sociétés depuis l'Antiquité, et le premier enregistrement de la consommation de marijuana (cannabis) remonte à au moins dix mille ans, dans lequel il ya des dossiers de culture et d'utilisation par diverses civilisations à travers le monde, étant le plus ancien en Asie et au Moyen-Orient, arrivant plus tard en Afrique, en Amérique et dans d'autres régions (ROSA; ROSA, 2018).

On estime qu'entre 167 et 315 millions de personnes âgées de 15 à 64 ans ont consommé des drogues illicites au cours de la dernière décennie, ce qui représente de 3,6 à 6,9 \% de la population mondiale. Dans ce contexte, la consommation de marijuana a augmenté à l'échelle mondiale, étant la drogue illicite la plus consommée dans le monde. On estime que la marijuana est consommée par 125 à 203 millions de personnes aujourd'hui, avec la prévalence la plus élevée en Afrique centrale et de l'Ouest (GARCIA, 2014).

RC: 80475

Disponível em: https://www.nucleodoconhecimento.com.br/sante/marijuana 
Au Brésil, environ 7,5 millions d'étudiants universitaires, répartis dans environ 2400 établissements utilisent l'herbe, et l'utilisation de cette substance est une pratique fréquente, très discutée par les médias laïques et analysée par certaines études scientifiques (GARCIA, 2014; FERNANDES et al., 2017).

Puisque la consommation de marijuana est de plus en plus fréquente dans notre environnement social, il est extrêmement important que des études soient faites afin d'élargir les connaissances sur ses effets chimiques, psychologiques et sociaux en raison de l'importance qu'ils présentent.

En outre, il ya eu des discussions ces dernières années sur les avantages et les inconvénients liés à la légalisation de cette drogue, et pour les professionnels et la population d'être en mesure de donner leur avis, il est nécessaire de comprendre d'abord comment la marijuana modifie le corps à court et à long terme (COUTINHO; ARAÚJO; GONTIÈS, 2004).

Dans ce contexte, ce travail est justifié par sa création afin d'affiner les connaissances existantes sur l'usage de la marijuana et de mettre à jour la société universitaire sur le processus de plaisir et de maladie auquel cette drogue est directement liée.

Cet examen vise à aborder les principaux aspects de la dépendance chimique par la consommation de marijuana, ainsi que les concepts sur les sensations agréables de l'utilisation de cette drogue et comment il contribue au désir de continuer son utilisation. II est également intéressant pour cet article de discuter des conséquences physiques et psychologiques que la marijuana provient de l'organisme caractérisant un processus de la maladie par son utilisation prolongée, contribuant à une meilleure information sur les raisons de ne pas utiliser cette drogue.

RC: 80475

Disponível em: https://www.nucleodoconhecimento.com.br/sante/marijuana 


\section{MATÉRIEL ET MÉTHODE}

La méthodologie utilisée dans la présente étude était une étude descriptive basée sur une revue de littérature. La recherche bibliographique a été effectuée par l'intermédiaire de la base de données Scielo et de la Bibliothèque virtuelle de santé (BVS). Pour rechercher les résumés portugais dans de telles bases, les termes suivants ont été utilisés: marijuana, plaisir et maladie. Après ce processus initial, les titres ont été lus, puis les résumés, pour sélectionner les publications qui ont intéressé la recherche, en fonction des exigences établies comme critères d'inclusion et d'exclusion. Cette recherche a eu comme critères d'inclusion des articles publiés dans la période de 2002 à 2020, en langue portugaise, qui abordent les thèmes liés à la marijuana et ses principaux effets physiologiques et pathologiques sur le corps humain, en ce qui concerne le corps et l'esprit. Les critères d'exclusion étaient des articles publiés dans une langue étrangère qui ne répondaient pas aux critères d'inclusion. Enfin, les articles sélectionnés ont été analysés et sont à l'origine de l'écriture du présent ouvrage.

\section{REVUE DE LITTÉRATURE ET DISCUSSION}

\subsection{CONCEPTS DE BASE LIÉS À LA MARIJUANA}

La marijuana est une herbe dont le nom scientifique est Cannabis. En latin Cannabis signifie chanvre, qu'il appelle la famille des plantes, et il existe trois types d'espèces de ce, étant sativa, indica et ruderalis (FERRARI, 2016). L'espèce, sativa et indica sont les plus utilisées, à la fois pour fumer, comme pour diverses applications dans la cuisine et la médecine (CORTEZ, 2009).

Pendant des siècles, la population a été en utilisant de l'herbe de marijuana à des fins médicinales, par exemple, les Chinois ont souligné les propriétés médicinales de la marijuana comme étant anticonvulsiant, apaisant et analgésique. Le potentiel de ses propriétés médicinales dans le traitement contre certaines maladies est déjà

RC: 80475

Disponível em: https://www.nucleodoconhecimento.com.br/sante/marijuana 
scientifiquement prouvé, nécessitant d'autres études pour analyser ses effets dans ce domaine (RIBEIRO; MARQUES, 2008).

Cannabis sativa contient environ 400 produits chimiques, dont au moins 60 alcaloïdes, connus sous le nom de cannabinoïdes. Le principal constituant psychoactif de la plante est le tétrahydrocannabiol (THC), l'un des composés de l'herbe, qui se compose également d'autres cannabinoïdes tels que le cannabidiol (CBD), le cannabinol (CBN) et le tétrahydrocanabivarin (THCV). Ceux-ci sont divisés en psychoactifs (delta-8-THC, delta-9-THC et 11-hydroxy-delta-9-THC) et non psychoactifs (cannabidiol et cannabinol). Delta-9-THC est connu pour être le plus puissant des cannabinoïdes (SOLOWIJ; PESA, 2008; CORTEZ, 2009).

\subsection{L’EFFET ET LE PLAISIR}

La principale façon d'utiliser l'herbe de marijuana est par le tabagisme et la biodisponibilité du THC lorsqu'il est fumé est d'environ $20 \%$. D'autres formes d'utilisation peuvent être effectuées par le haschisch qui peut également être fumé et a des concentrations plus élevées de THC, et hashoil ou huile de cannabis, qui est la forme liquide et la plus puissante de la drogue intraveineuse. Lorsqu'elle est inhalée, la fumée générée par l'herbe brûlée atteint les alvéoles pulmonaires, pénètre dans la circulation sanguine et atteint le cerveau en quelques minutes (CORTEZ, 2009).

Les taux d'absorption orale sont plus élevés (90 à $95 \%$ ) (30 à 45 minutes) par rapport à l'absorption pulmonaire (50\%). Les effets pharmacologiques par absorption pulmonaire peuvent prendre entre 5 et 10 minutes pour commencer (SOLOWIJ; PESA, 2008; CORTEZ, 2009).

Les effets de pointe causés par le médicament se produisent en 30 minutes et se terminent après environ deux ou trois heures (CORTEZ, 2009).

Les cannabinoïdes sont déposés principalement dans les organes riches en tissus adipeux, tels que le cerveau et les testicules, et donc certains utilisateurs peuvent

RC: 80475

Disponível em: https://www.nucleodoconhecimento.com.br/sante/marijuana 
présenter des signes et des symptômes d'intoxication jusqu'à 12-24h en raison de la lente libération de ces substances par les adipocytes (SOLOWIJ; PESA, 2008).

La demi-vie du THC peut varier de $20 \mathrm{~h}$ à 10 à 13 jours et l'élimination totale peut prendre jusqu'à 30 jours. Ainsi, si un individu consomme de la marijuana à plusieurs reprises à des intervalles plus petits que celui-ci, la concentration de THC dans son sang augmentera, causant des effets plus intenses (SOLOWIJ; PESA, 2008; CORTEZ, 2009).

Les cigarettes de marijuana, largement répandues, bien qu'interdites dans certains pays comme le Brésil, sont également connues sous le nom de « based », consistant en environ 0,3 à $1 \mathrm{~g}$ de marijuana avec une concentration de 1 à $15 \%$ de delta-9THC (2,5 à $150 \mathrm{mg}$ de THC). Dans ce contexte, on observe que la production d'effets euphoriques se produit avec une concentration minimale de $1 \%$ de THC ou 1 cigarette de 2 à $5 \mathrm{mg}$. Et contrairement à ce que beaucoup pensent, les symptômes d'intoxication apparaissent déjà quelques minutes après utilisation (RIBEIRO; MARQUES, 2008; SOLOWIJ; PESA, 2008).

En ce qui concerne les effets de la marijuana, dans certaines études les symptômes liés à l'utilisation récréative et l'abus de cette drogue sont signalés. Cependant, au niveau universitaire, les effets de la consommation de marijuana peuvent être divisés en symptômes aigus et chroniques. Les effets aigus sont classés comme euphoriques (augmentation du désir sexuel, sensation de ralentissement du temps, augmentation de la confiance en soi et de la grandeur, rires non motivés, hilarité, relaxation et perception accrue des couleurs, des sons, des textures et du goût), effets physiques (tachycardie, hyperémie conjonctivale, sécheresse de la bouche, hypothermie, étourdissements, retard psychomoteur, incoordination motrice, diminution de l'acuité visuelle, augmentation de l'acuité auditive, bronchodilation, augmentation de l'appétit, toux et mydriase) et effets psychiques (dépersonnalisation), la disréalisation, la dépression, l'hallucination, l'illusion, la somnolence, les troubles de la concentration et de la mémoire à court terme, les crises de panique et la paranoïa) (SOLOWIJ; PESA, 2008; CORTEZ, 2009).

$\mathrm{RC}: 80475$

Disponível em: https://www.nucleodoconhecimento.com.br/sante/marijuana 


\subsection{ABUS ET MALADIE}

Certains auteurs induisent que la consommation de substances psychoactives a traversé les temps et les cultures, étant utilisé dans les rituels religieux, comme une méthode thérapeutique et dans la recherche du plaisir, en mettant l'accent sur I'hédonisme comme une référence dans ce processus (COUTINHO; ARAÚJO; GONTIÈS, 2004).

À court terme, on peut observer les effets nocifs de la consommation de marijuana, comme les déficits moteurs, lors de l'exécution de tâches telles que la conduite, par exemple; et les problèmes cognitifs, la concentration et la mémoire et, par conséquent, les difficultés d'apprentissage. D'autre part, l'utilisation à long terme peut être marquée par la toux chronique, l'immunité altérée, les niveaux réduits de testostérone et le développement de maladies mentales telles que la schizophrénie, la dépression, les crises de panique, l'anxiété, l'irritabilité, la démotivation par la vie, les hallucinations et la dépersonnalisation. Plus la dose consommée est élevée, plus les effets et les taux de maladies seront intenses. La dépendance à la consommation de marijuana se produit dans la mesure de sa consommation. Les effets indésirables comprennent l'amnésie, le manque d'attention, les problèmes moteurs, la génération de crises de dépendance et de sevrage (RIBEIRO; MARQUES, 2008; SOLOWIJ; PESA, 2008).

Certaines études montrent un dysfonctionnement cognitif chez les consommateurs de cannabis pendant l'intoxication, plusieurs heures après l'acte de le fumer, après quelques jours et des dommages durables pendant plus d'un mois après l'arrêt de la consommation. La chronicité des altérations cognitives dépend de l'organisme, de la dose et de la périodique que l'individu utilise de l'herbe, mais il est important de souligner que depuis la première utilisation du médicament et après le début de l'action du THC, ces pertes psychiques se manifestent déjà (RIBEIRO; MARQUES, 2008).

RC: 80475

Disponível em: https://www.nucleodoconhecimento.com.br/sante/marijuana 
Les relations entre la performance cognitive et la fréquence de la consommation de cannabis peuvent indiquer un effet résiduel d'intoxication aiguë ou chronique qui se dissiperait probablement avec l'arrêt de la consommation. Certaines études rapportent que le rétablissement de la fonction cognitive n'est pas entièrement élucidé, mais dans certaines sources, il est constaté que la récupération peut se produire après 1 mois d'abstinence ou ne se produit pas dans 1 mois ou le rétablissement peut être seulement partielle (RIBEIRO; MARQUES, 2008; SOLOWIJ; PESA, 2008).

L'utilisation de la marijuana peut également entraîner des changements structurels dans le cerveau, tels que la réduction des volumes de l'hippocampe et l'amygdale, et il ne peut pas encore être affirmé que ces effets sont réversibles. Mais grâce à ce processus, on peut comprendre la vraie raison pour laquelle l'affaiblissement cognitif est plus fréquemment détecté dans la mémoire, la fonction exécutive, l'attention et le contrôle inhibiteur (SOLOWIJ; PESA, 2008).

Dans ce contexte, il est intéressant de noter que le sujet qui consomme de la marijuana peut ou non avoir une raison apparente d'effectuer une telle utilisation, et parmi les principaux facteurs stimulants pour la consommation de la drogue peut être mentionné: échapper aux problèmes, la curiosité, la recherche de plaisirs et d'influence sociale.

\section{CONCLUSION}

On peut observer que la consommation de marijuana implique une série d'effets aigus et chroniques, ainsi que résiduels, liés au mécanisme d'action du THC. En outre, les utilisateurs de ce médicament ont souvent des problèmes qui affectent leur vie familiale, professionnelle et sociale.

Dans ce contexte, il est intéressant d'observer que le sujet qui consomme de la marijuana peut ou non avoir une raison apparente d'effectuer l'utilisation, mais ce qui suscite le désir de promouvoir des études sur le pont entre le plaisir et la maladie est

$\mathrm{RC}: 80475$

Disponível em: https://www.nucleodoconhecimento.com.br/sante/marijuana 
ce qui est derrière la recherche de la substance, un fait qui est au-delà des yeux de l'utilisateur et le conscient.

Dans la présente étude, certaines preuves sont démontrées qui peuvent expliquer les raisons pour lesquelles les individus choisissent de fumer de la marijuana. Le plus souvent, les effets aigus de l'euphorie, le plaisir et la relaxation sont souhaités, en revanche, il est observé que les dommages associés à cette utilisation finissent par surmonter les avantages illusoires que le médicament offre, conduisant ainsi au plaisir de devenir une maladie.

\section{RÉFÉRENCES}

CORTEZ, P. Possíveis efeitos cognitivos e psicomotores em usuários crônicos de Cannabis. 2009.

COUTINHO, M. P. L.; ARAÚJO, L. F.; GONTIÈS, B. Uso da maconha e suas representações sociais: estudo comparativo entre universitários. Psicologia em Estudo, Maringá, v. 9, n. 3, p. 469-477, set./dez. 2004.

FERNANDES, T. F.; MONTEIRO, B. M. M.; SILVA, J. B. M.; OLIVEIRA, K. M.; VIANA, N. A. O.; GAMA, C. A. P.; GUIMARÃES, D. A. Uso de substâncias psicoativas entre universitários brasileiros: perfil epidemiológico, contextos de uso e limitações metodológicas dos estudos. Cad. Saúde Colet., 2017, Rio de Janeiro, 25 (4): 498-507.

FERRARI, C. R. Cannabis. Universidade de São Paulo. Instituto de Física de São Carlos - Psicologia da Educação - SLC 0631. Licenciatura em Ciências Exatas. 2016. $15 p$.

GARCIA, F. D. Manual de abordagem de dependências químicas. ED. Utopika, 2014, 386p.

RC: 80475

Disponível em: https://www.nucleodoconhecimento.com.br/sante/marijuana 
RIBEIRO, M; MARQUES, A.C. P. R. Projeto diretrizes: Abuso e dependência Maconha. 2008.

ROSA, P.; ROSA, M. G. Políticas sobre cannabis: um estudo comparativo sobre os modelos da Espanha. Geographia Opportuno Tempore Universidade Estadual de Londrina EISSN: 2358-1972, Volume 4, Número 1, 2018 Uruguai e Colorado/EUA, 2018.

SOLOWIJ, N,; PESA, N. Anormalidades cognitivas no uso da cannabis. 2010.

Soumis: Mars, 2021.

Approuvé: Mars, 2021.

RC: 80475

Disponível em: https://www.nucleodoconhecimento.com.br/sante/marijuana 